\title{
Prohibir el mestizaje con chinos: solicitudes de amparo, Sonora, 1921-1935
}

por

\section{Kif Augustine-Adams}

J. Reuben Clark Law School, Brigham Young University

En el estado de Sonora la población china enfrentó una manifiesta discriminación expresada en la Ley 31 de 1923. Esta Ley prohibía el matrimonio y la mestización entre hombres chinos y mujeres mexicanas. Por medio de peticiones de amparo, parejas mexicano-chinas desafiaron esta legislación, llegando con los procesos legales hasta la Suprema Corte de Justicia. En los años 1924 y 1925, jueces federales en Sonora aplicaron los principios de la Constitución y de la legislación federal para proteger a la población china. Las peticiones de amparo demuestran tanto los desafios como las posibilidades que el derecho otorgó a los residentes de México en las primeras décadas del siglo XX.

Palabras clave: México; chinos; discriminación; uniones matrimoniales; procesos legales.

En diciembre de 1923, el Congreso del estado de Sonora, México, aprobó una ley contra el mestizaje. La Ley 31 prohibía «el matrimonio de mujeres mexicanas con individuos de raza china, aunque obtengan carta de naturalización mexicana» ${ }^{1}$. Prohibía también otras relaciones íntimas entre chinos y mexicanas ${ }^{2}$. En Sonora, el prejuicio contra los chinos existía desde hacía mucho tiempo; sin embargo, la Ley 31 inaugura un periodo de mayor discriminación contra la población china ${ }^{3}$. Las parejas chino-

1 Sonora, Ley número 31, 13 de diciembre de 1923, Espinoza, 1932: 35.

2 Idem.

3 Véase Hu-Dehart, 1985: 195-211. González Oropeza, 1997: 47-56. Liam, LXXIX / 1 (California, 2010): 50-85. Schiavone Camacho, LXXVIII / 4 (California, 2009): 545-77. Augustine-Adams, XXVII / 1 (Illinois, 2009): 113-44. 
mexicanas no tardaron en defenderse: desafiaron esa Ley en tribunales federales, pidiendo amparo contra su aplicación ${ }^{4}$. Por medio de estas solicitudes de amparo, mexicanas y chinos usaron al poder judicial federal para combatir la discriminación. Teniendo en cuenta las políticas antichinas en Sonora, esta estrategia legal fue sorprendentemente exitosa, aunque por poco tiempo.

Las solicitudes contra la aplicación de la Ley 31 empezaron en 1924, justo cuando el aparato judicial se reestructuraba después de los trastornos que trajo consigo la Revolución de 1910. La Constitución de 1917 no tenía ni diez años de vigencia, y la aplicación jurídica de sus principios estaba todavía en ciernes. El éxito inicial de las peticiones contra la Ley 31 representa un momento breve pero brillante en la historia del constitucionalismo y de la administración de justicia en México. Los jueces federales en Sonora, y en particular el juez Arsenio Espinosa, se aferraron a los principios de la Constitución y de la legislación federal para proteger a una minoría despreciada por el poder político de una mayoría cada vez más agresiva e intolerante.

Finalmente, el amparo federal que otorgó el juez Espinosa no prevaleció, ni logró proteger a los chinos de la expulsión de Sonora entre 1931 y 1932, expulsión violenta que fue patrocinada por el gobierno estatal ${ }^{5}$. No obstante,

${ }^{4}$ Como explica Ignacio Burgoa, «el juicio de amparo surgió a la vida jurídica de México merced al impuso social... de proteger las garantías individuales o los llamados "derechos del hombre", principalmente, es decir, la esfera del gobernado contra cualquier acto del orden público que afectase o amenazase su integridad...», Burgoa, 1968: 26. Todas las solicitudes de amparo a que se refiere este artículo se encuentran en la colección de Amparo Civil, 1900-1943, Archivos del Juzgado Quinto de Distrito, Casa de la Cultura Jurídica de la Suprema Corte de la Nación, Hermosillo, Sonora, México. En 2011, no había inventario oficial de la colección y en muchas de las cajas la información está incompleta. Por ejemplo, en las cajas de 1924, faltan los amparos de los número 461 al 478. En las cajas de 1925, las solicitudes empiezan en mayo faltando todas las solicitudes presentadas de enero a fines de abril de ese año. Este artículo se basa en las solicitudes de amparo que había en la colección en 2005 y 2011.

5 Réñique, 2003: 231-89. Frente al tribunal federal de Hermosillo, varios hombres chinos describieron cómo la policía y otras autoridades gubernamentales los habían llevado a la frontera entre Arizona y Sonora, amenazándolos con violencia y los habían forzado a cruzar. Véase, por ejemplo, Petición de Amparo no. 82, Francisco Ley et al., 11 de agosto de 1932, Casa de la Cultura Jurídica de la Suprema Corte de la Nación, Hermosillo, Sonora, México (CCJSCN), Archivos del Juzgado Quinto de Distrito, Amparo Civil, 1900-1943, y Petición de Amparo no. 77, Agustín Chang, 16 de agosto de 1932 (CCJSCN), Archivos del Juzgado Quinto de Distrito, Amparo Civil, 1900-1943. La expulsión forzosa de los chinos de Sonora a Arizona causó consternación en la frontera y tensiones diplomáticas entre los Estados Unidos y México. Véase Arizona Daily Star, Arizona, 19 de marzo de 1932, editorial; W. Doak, Secretaría del trabajo de los Estados Unidos a Henry L. Stimson, Secretaría de 
las peticiones de amparo contra de Ley 31 muestran tanto las limitaciones como las posibilidades que el derecho ofrecía a individuos excluidos por cuestiones raciales.

\section{Peticiones DE AMPARO}

En enero de 1924, solo un mes después de la promulgación de la Ley 31, Manuel Yee presentó la primera solicitud de amparo federal ${ }^{6}$. Yee había nacido en China pero se había naturalizado mexicano ${ }^{7}$. Alegó que el presidente municipal de Nogales, Walterio Pesqueira, lo había encarcelado e impuesto una multa de 100 pesos por vivir con Rosario Hernández, aunque Yee sostenía que se había separado de Hernández «al principio de diciembre», antes de la promulgación de la ley8.

Posteriormente, y en una rápida sucesión se presentaron solicitudes de amparo con alegatos similares. Ramón Gan objetó que se le aplicara la Ley 31 pues se había casado con su esposa, Lucia Jaime de Gan, el 23 de agosto de 1922 en Douglas, Arizona, más de dos años antes de la promulgación de la Ley $31^{9}$. Pablo Wong y Filomena Valdez buscaron ampararse contra la multa que tenían que pagar para salir de la cárcel de Cananea, arguyendo que «hace más de ocho años que venimos haciendo vida marital, sin escándalos públicos y sin ofender a la moral en lo más mínimo» ${ }^{10}$. Carlos Fong y Esperanza Parra también tenían ocho años como pareja cuando solicitaron amparo contra la multa y el encarcelamiento que sufrieron por vivir juntos ${ }^{11}$. Julia Delgado y Francisco Gim solicitaron amparo contra el

Estado de los Estados Unidos, 16 de marzo de 1932, Estados Unidos de América, Archivos Nacionales (en adelante AN), RG59, M1370, 812.504/1281; Bartley F. Yost, Consul, a Henry L. Stimson, Secretaria de Estado, 21 de marzo de 1932, AN, RG59, M1370, 812.504/1282.

6 Solicitud de amparo 391A, Manuel Yee, contra prisión y multa de \$100 bajo Ley 31, 15 de enero de 1924 (CCJSCN), Archivos del Juzgado Quinto de Distrito, Amparo Civil, 1900-1943.

7 Idem.

8 Idem.

9 Solicitud de amparo 350A, Ramón Gan y Lucia Jaime de Gan, contra la aplicación de la Ley 31, 29 de febrero de 1924, CCJSCN, Archivos del Juzgado Quinto de Distrito, Amparo Civil, 1900-1943.

10 Solicitud de amparo 364A, Pablo Wong y Filomena Valdez, contra la aplicación de la Ley 31, 6 de marzo de 1924, CCJSCN, Archivos del Juzgado Quinto de Distrito, Amparo Civil, 1900-1943.

11 Solicitud de amparo 363B, Carlos Fong, contra multa de $\$ 100$ con apoyo en la Ley 31 del Estado, 6 de marzo de 1924, CCJSCN, Archivos del Juzgado Quinto de Distrito, Am- 
juez del Registro Civil que se rehusó a darles licencia para casarse ${ }^{12}$. En 1929, Carlos Wong Sun hizo lo mismo después de que el juez del Registro Civil en Cucurpé se rehusara a levantar el acta para que pudiera casarse con Juana Ramírez ${ }^{13}$.

Las solicitudes de amparo contra la aplicación de la Ley 31 formaban parte de una estrategia legal más amplia contra la discriminación en Sonora. Además de sus quejas contra la Ley 31, entre 1921 y 1935, algunos chinos presentaron solicitudes de amparo ante diversas leyes, reglamentos y acciones gubernamentales. Pidieron amparo contra la aplicación de la Ley 27 de 1923, que creaba barrios exclusivos donde todos los chinos debían mudarse ${ }^{14}$; contra la aplicación selectiva de reglamentos de sanidad y moralidad ${ }^{15}$; contra la imposición selectiva de impuestos ${ }^{16}$; contra leyes de trabajo que estipularon que los chinos emplearan en sus negocios solo a mexicanos por nacimiento, norma que fue impuesta para impedir la contratación de chinos que se habían naturalizado mexicanos ${ }^{17}$; $\mathrm{y}$, finalmente, los amparos se interpusieron contra

paro Civil, 1900-1943; Petición de amparo 363B, Esperanza Parra, contra prisión y aplicación de la Ley 31, 6 de marzo de 1924, CCJSCN, Archivos del Juzgado Quinto de Distrito, Amparo Civil, 1900-1943.

12 Solicitud de amparo 12, Francisco Gin y Julia Delgado, aplicación de la Ley número 31, 12 de febrero de 1926, CCJSCN, Archivos del Juzgado Quinto de Distrito, Amparo Civil, 1900-1943. El apellido Gim se transcribe del chino al español en varias maneras. En este trabajo utilizo Gim porque es la forma que usaba la familia, aunque en las notas sigo la ortografía original de la fuente utilizando tanto Gim, Gin o Hing.

13 Solicitud de amparo 10, Carlos Wong Sun, contra aplicación de la Ley 31, 10 de febrero de 1929, CCJSCN, Archivos del Juzgado Quinto de Distrito, Amparo Civil, 1900-1943.

14 Véase, por ejemplo, Solicitud de amparo 329A, Felipe Chon, José Wontui, Gregorio Chan, Samuel Cinco, Wong Lee, Alberto Chan Po, Adolfo Chan y Sam Wo, contra Ley 27, 12 de febrero de 1924, CCJSCN, Archivos del Juzgado Quinto de Distrito, Amparo Civil, 19001943 (concediendo amparo); Petición de amparo 336A, Antonio Bonio, Alejandro Yee y coagraviados, contra Ley 27, 25 de febrero de 1924, CCJSCN, Archivos del Juzgado Quinto de Distrito, Amparo Civil, 1900-1943 (concediendo amparo); Petición de amparo 344A, Manuel L. Chew, Mariano M. Wo y coagraviados, contra aplicación de la Ley No. 27, 25 de febrero de 1924, CCJSCN, Archivos del Juzgado Quinto de Distrito, Amparo Civil, 1900-1943.

15 Por ejemplo, Petición de amparo 579, Luis Madero, Gustavo Chan, Antonio Chao, Lorenzo Fuguay y coagraviados contra imposición de una multa de $\$ 10.00$ por infracción al Reglamento de Sanidad, 1925, CCJSCN, Archivos del Juzgado Quinto de Distrito, Amparo Civil, 1900-1943.

16 Por ejemplo, Petición de amparo 415A, Antonio Won, Juan Wong, Francisco Chao y Antonio Wong, contra prisión y multa de $\$ 300$ pesos impuestos, 1925, CCJSCN, Archivos del Juzgado Quinto de Distrito, Amparo Civil, 1900-1943.

17 Por ejemplo, Petición de amparo 49, Félix Cinco y socios, contra Ley 89 de Estado y aplicación de la Ley de trabajo, 1931, CCJSCN, Archivos del Juzgado Quinto de Distrito, Amparo Civil, 1900-1943 (sobreseyendo la petición); Petición de amparo 71, Lorenzo Toy y 
órdenes de expulsión del país ${ }^{18}$. Esta estrategia de acudir a tribunales federales fue la misma que emplearon los chinos en Estados Unidos cuando enfrentaron la legislación discriminatoria promulgada tanto por los estados como por el gobierno federal en la década de los años 1880. Al igual que en Sonora, en Estados Unidos tuvo resultados desiguales ${ }^{19}$.

Para contextualizar los desafíos a la Ley 31 en Sonora, la Tabla 1 presenta por año: 1) el número total de solicitudes de amparo presentadas en los juzgados federales en Sonora; 2) el número total de solicitudes de amparo presentadas por chinos; y 3) el número de solicitudes presentadas contra la Ley 31 específicamente. La Tabla 2 identifica las solicitudes de amparo contra la Ley 31 por año, juez, y el tipo de resolución ${ }^{20}$.

socios, sobre aplicación de la Ley de Trabajo, Ley 89 del Estado, 1931, CCJSCN, Archivos del Juzgado Quinto de Distrito, Amparo Civil, 1900-1943 (sobreseyendo la petición); Petición de amparo 93, Fu Pau Hermanos y compañia, contra imposición de multa de \$500 y Leyes 89 y 106 del Estado, 17 de agosto de 1931, CCJSCN, Archivos del Juzgado Quinto de Distrito, Amparo Civil, 1900-1943.

18 Por ejemplo, Petición de amparo 104, José Luy por Josefina Bustamente de Luy, contra expulsión, 1932, CCJSCN, Archivos del Juzgado Quinto de Distrito, Amparo Civil, 19001943.

19 Véase, por ejemplo, Supreme Court of the United States, 1886: 356-374. El caso de Yick Wo afirmó que toda persona, fuera ciudadana o no, estaba bajo la protección de la enmienda 14. Véase también Supreme Court of the United States, 1889: 581-611. En The Chinese Exclusion Case, la Suprema Corte de los Estados Unidos ratificó que el Congreso nacional tenía plena autoridad para excluir a los no ciudadanos del país. Para un análisis exhaustivo de las estrategias legales que empleó la población china en los Estados Unidos contra la discriminación a finales del siglo XIX, vease Salyer, 1995.

20 Todos los casos se encuentran en el CCJSCN, Archivos del Juzgado Quinto de Distrito, Amparo Civil, 1900-1943. 
TABla 1. Solicitudes DE AMPARO PRESENTADAS POR CHINOS EN JUZGADOS FEDERALES, SONORA, 1921-1935

\begin{tabular}{|c|c|c|c|c|c|c|}
\hline \multirow[b]{2}{*}{ Año } & \multirow{2}{*}{$\begin{array}{l}\text { Solicitudes de } \\
\text { amparo } \\
\text { promovidas en } \\
\text { juzgados } \\
\text { federales en } \\
\text { Sonora }\end{array}$} & \multicolumn{2}{|c|}{$\begin{array}{l}\text { Solicitudes de amparo } \\
\text { promovidas por chinos }\end{array}$} & \multicolumn{3}{|c|}{$\begin{array}{c}\text { Solicitudes de amparo promovidas } \\
\text { contra la Ley } 31^{*}\end{array}$} \\
\hline & & Total & $\begin{array}{c}\text { Por porcentaje } \\
\text { de solicitudes } \\
\text { promovidas en } \\
\text { juzgados } \\
\text { federales en } \\
\text { Sonora }\end{array}$ & Total & $\begin{array}{l}\text { Por porcentaje } \\
\text { de solicitudes } \\
\text { promovidas en } \\
\text { juzgados } \\
\text { federales en } \\
\text { Sonora }\end{array}$ & $\begin{array}{c}\text { Por porcentaje } \\
\text { de solicitudes } \\
\text { promovidas } \\
\text { por chinos }\end{array}$ \\
\hline 1921 & 30 & 1 & $3 \%$ & & & \\
\hline 1922 & 23 & & & & & \\
\hline 1923 & 85 & 6 & $7 \%$ & & & \\
\hline 1924 & 173 & 89 & $51 \%$ & 20 & $11 \%$ & $22 \%$ \\
\hline 1925 & 76 & 12 & $16 \%$ & 3 & $4 \%$ & $25 \%$ \\
\hline 1926 & 103 & 9 & $9 \%$ & 4 & $4 \%$ & $44 \%$ \\
\hline 1927 & 67 & 2 & $3 \%$ & & & \\
\hline 1928 & 96 & 3 & $3 \%$ & & & \\
\hline 1929 & 64 & 2 & $3 \%$ & 2 & $3 \%$ & $100 \%$ \\
\hline 1930 & 77 & & & & & \\
\hline 1931 & 122 & 27 & $22 \%$ & 1 & $.8 \%$ & $4 \%$ \\
\hline 1932 & 74 & 13 & $17 \%$ & 1 & $1.3 \%$ & $8 \%$ \\
\hline 1933 & 97 & 1 & $1 \%$ & & & \\
\hline 1934 & 80 & & & & & \\
\hline 1935 & 52 & 1 & $2 \%$ & & & \\
\hline Total & 1.219 & 166 & $14 \%$ & 31 & $2.5 \%$ & $19 \%$ \\
\hline
\end{tabular}

* Las solicitudes de amparo contra la Ley 31 incluyen solicitudes presentadas por mujeres mexicanas con sus compañeros chinos, así como dos peticiones presentadas por mujeres mexicanas de forma individual. 
Tabla 2. Solicitudes de Amparo CONTRA la Ley 31 POR AÑo, JUEZ, Y RESOLUCIÓN, SONORA, 1921-1935

\begin{tabular}{|l|l|l|l|l|}
\hline \multirow{2}{*}{ Año } & \multirow{2}{*}{ Juez } & \multicolumn{2}{c|}{ Resolución } \\
\cline { 3 - 5 } & & amparado & negado & sobreseido $^{21}$ \\
\hline 1921 & & & & \\
\hline 1922 & & & & \\
\hline 1923 & & & & \\
\hline 1924 & Arsenio Espinosa & 19 & & 1 \\
\hline 1925 & Cayetano Masse & 2 & & \\
\hline & Luís Bazdresch & 1 & & \\
\hline 1926 & Joaquín Ruíz & & & \\
\hline 1927 & & & & \\
\hline 1928 & & & & \\
\hline 1929 & Joaquín Silva & & & \\
\hline 1930 & & & & \\
\hline 1931 & Manuel Gómez Lomelí & & & \\
\hline 1932 & Manuel Gómez Lomelí & & & \\
\hline 1933 & & & & \\
\hline 1934 & & & & \\
\hline 1935 & & & & \\
\hline Total & & & & \\
\hline
\end{tabular}

Los quejosos presentaron 20 de las 31 demandas de amparo (65\%) en 1924, año en el que las solicitudes chinas constituyeron más de la mitad de los juicios de amparo en Sonora. El juez Arsenio Espinosa oyó todas las solicitudes promovidas por chinos en 1924, incluyendo las veinte promovidas contra la Ley $31^{22}$. Concedió todos los amparos contra la Ley 31 que se le presentaron, menos una que sobreseyó cuando los demandantes salieron de la cárcel y no prosiguieron la demanda ${ }^{23}$. En 1925, dos jueces distintos, Caye-

21 Sobreseído significa que el juez desechó el caso por no cumplir con algún procedimiento o requisito, sin considerar los méritos o la pertinencia de la petición.

22 La gran mayoría de los otros casos en 1924 eran peticiones contra la aplicación de la Ley 27 que creaba barrios chinos. Véase la nota 14 para ejemplos.

23 Solicitud de amparo 326A, José Sujo y Rafael Yuen, contra prisión y multa bajo la Ley 31, 1924, CCJSCN, Archivos del Juzgado Quinto de Distrito, Amparo Civil, 1900-1943. 
tano Masse y Luis Bazdresch, oyeron tres casos contra la aplicación de la Ley 31 y concedieron el amparo en todos ellos ${ }^{24}$.

En 1926, el juez Joaquín Ruiz rompió con el precedente informal que habían creado los jueces Espinosa, Masse y Bazdresch. Negó dos de las cuatro solicitudes que se le presentaron y sobreseyó dos más por fallas en el proceso, ya que el demandante en un caso no ratificó formalmente el proceso que inició por telegrama, y en otro abandonó su queja ${ }^{25}$. En cambio, de los pocos recursos que se presentaron contra la Ley 31 después de 1926, todos fracasaron en el tribunal de primera instancia. Estos incluyeron dos solicitudes presentadas en 1929, una por Francisco Gim y otra por Carlos Wong Sun. Ambos quejosos apelaron a la Suprema Corte de Justicia de la Nación.

Los siguientes recursos de amparo contra la Ley 31 siguieron la misma fórmula. Con mínimas variaciones, todas las solicitudes alegaron que se violaban varios artículos de la Constitución de 1917, aunque las peticiones simplemente identifican al artículo sin describir el derecho en cuestión: el artículo $1 .^{\circ}$ que garantizaba los derechos individuales; el artículo 14 que prohibía la privación de «la vida, de la libertad o de propiedades, posesiones o derechos» sin un juicio apegado a las formalidades esenciales del procedimiento; el artículo 16 que establecía que nadie podía ser molestado en su «persona, familia, domicilio, papeles y posesiones» sino «en virtud de mandamiento escrito de la autoridad competente, que funde y motive la causa legal del

24 Solicitud de amparo 561, Roberto H. Chan, por haberse negado el funcionario del Registro civil a efectuar el matrimonio con la señorita Carmen Figueroa, 12 de mayo de 1925, CCJSCN, Archivos del Juzgado Quinto de Distrito, Amparo Civil, 1900-1943; Solicitud de amparo 586, Ramón Chan, por haberse negado el funcionario del Registro civil a efectuar la presentación matrimonial con la señorita Anita Duran, 24 de julio de 1925, CCJSCN, Archivos del Juzgado Quinto de Distrito, Amparo Civil, 1900-1943 (concediendo el amparo); Solicitud de amparo 602, Manuel H. Fu, por haberse negado el funcionario del Registro civil a efectuar la presentación matrimonial con Amelia Domínguez, 8 de septiembre de 1925, CCJSCN, Archivos del Juzgado Quinto de Distrito, Amparo Civil, 1900-1943 (concediendo el amparo). Luís Bazdresch llegó a ser ministro de la Suprema Corte de México en 1934. Véase Suprema Corte de Justicia de la Nación, 2002: 63-66.

25 Solicitud de amparo 12, Francisco Gin y Julia Delgado, aplicación de la Ley número 31, 12 de febrero de 1926, CCJSCN, Archivos del Juzgado Quinto de Distrito, Amparo Civil, 1900-1943; Solicitud de amparo 23, Miguel Wong, contra aplicación de la Ley 31, 6 de marzo de 1926, CCJSCN, Archivos del Juzgado Quinto de Distrito, Amparo Civil, 19001943; Solicitud de amparo 59, Fom Lim, 15 de julio de 1926, CCJSCN, Archivos del Juzgado Quinto de Distrito, Amparo Civil, 1900-1943; Solicitud de amparo 71, Jesús Sujo, contra impedirsele contraer matrimonio, 10 de agosto de 1926, CCJSCN, Archivos del Juzgado Quinto de Distrito, Amparo Civil, 1900-1943. 
procedimiento»; el artículo 21 que establecía que la imposición de penas era propia y exclusiva del poder judicial; y el artículo 30(II-B) que permitía a los extranjeros naturalizarse mexicanos. Además de los argumentos constitucionales, muchos de los quejosos citaron el Tratado de Amistad, Comercio y Navegación de 1899 entre México y China como fundamento del amparo. Las solicitudes de amparo variaron de acuerdo a los detalles efectivos sobre dónde, cuándo y cómo los funcionarios del gobierno habían intentado aplicar la Ley 31 contra de los quejosos.

\section{LA NATURALEZA DE AMPARO}

El amparo tiene una historia compleja y conflictiva en el derecho mexicano ${ }^{26}$. Tanto antes como después de la Revolución de 1910, los especialistas debatieron en torno a cuáles eran los derechos que el amparo debía proteger - derechos naturales, derechos constitucionales y otros derechos positivosy sobre el mecanismo mediante el cual debía impartirse protección ${ }^{27}$. En todo caso, el fallo de un juez otorgando un amparo solo protegía a los solicitantes ${ }^{28}$. Al amparar un juez a un quejoso, se suspendía la aplicación de la ley en el caso específico, no se invalidaba la ley ${ }^{29}$. El mecanismo de revisión judicial que originó en Estados Unidos en el caso Marbury v. Madison ${ }^{30}$ — autorizando a los tribunales a derogar leyes que se juzgaban anti-constitucionales - no arraigó en el sistema judicial de México, aunque la idea sí estuvo presente ${ }^{31}$. Ignacio Luis Vallarta, presidente de la Suprema Corte de la Nación entre 1878-1882 y jurista prominente, promovió enérgicamente algún tipo de revisión judicial al estilo de Marbury ${ }^{32}$. Al mismo tiempo, Vallarta publicó sentencias detalladas que recuerdan las formas del common-law anglo-americano, lo que podría facilitar que se recurriera a ellas como precedentes en futuras controversias judiciales ${ }^{33}$. Sin embargo, el compromiso de Vallarta con la revisión judicial y la forma en que presentaba sus opiniones no prevalecieron. En cambio, la jurisprudencia y el discurso legal mexicanos rechazaron la

26 Véase Burgoa, 1968: 280. Zamora, Cossío, Pereznieto, Roldán-Xopa, y López, 2004: 261-63.

27 Véase, por ejemplo, Hale, 2008: 37-38, 40-42, 169-170.

28 Burgoa, 1968: 280. Zamora, Cossío, Pereznieto, Roldán-Xopa, y López, 2004: 214.

29 Constitución de 1917, artículo 107(I).

30 Supreme Court of the United States, 1803: 137-139.

31 Véase Mirow, XXXV (Hastings, California, 2007-08): 41-117.

32 Vallarta, 1881-1883: t. 3: 383.

33 Vallarta, 1881-1883. 
teoría de la revisión judicial y reafirmaron la tradición del derecho civil en la que el papel del juez quedó muy acotado, particularmente después de la Revolución de $1910^{34}$.

En la tradición del derecho civil, un amparo por sí solo, no valía como precedente para otra solicitud de amparo; constituía una tesis aislada ${ }^{35}$. El artículo 107(I) de la Constitución de 1917, conocido como la fórmula Otero, indicaba que al concederse el amparo, el único beneficiado debía ser el solicitante «sin hacer una declaración general respecto de la ley o acto que la motivare ${ }^{36}$, construcción muy parecida a la de los artículos 101 y 102 de la Constitución de 1857. Aunque la fórmula Otero limitaba el valor formal de un solo fallo como precedente, este podía constituirse como tal a nivel informal. La reiteración de los criterios emitidos en un fallo crearía así jurisprudencia $^{37}$. Al recibir en rápida sucesión numerosas solicitudes de amparo contra la misma ley, basadas en datos similares, era posible que los fallos de los tribunales presentaran una uniformidad relativa ${ }^{38}$. Así lo demuestra el destino de las solicitudes de amparo presentadas por chinos en Sonora.

\section{Los fallos DEL JUEZ ARSENIO EsPinOSA}

Como se ha mencionado, Manuel Yee presentó la primera solicitud de amparo contra la Ley 31 en enero de 1924, a menos de un mes de su promulgación. Arsenio Espinosa, el juez federal del estado de Sonora, falló sobre la solicitud de Yee, primera de veinte solicitudes similares que atendería ${ }^{39}$. Con

34 Hale, XVIII / 2 (Illinois, USA, 2000): 257-279, 264.

35 Zamora, Cossío, Pereznieto, Roldán-Xopa y López, 2004: 85, 214. Es cierto que tesis aisladas, por lo menos al nivel de la Suprema Corte y en la actualidad en los tribunales colegiados de circuito, pueden llegar a ser jurisprudencia obligatoria o ser un precedente formal. Como lo explica Burgoa y citando la Ley de Amparo, para ser jurisprudencia de la Suprema Corte se requiere «dos condiciones legales, a saber: que aquella se establezca en cinco ejecutorias o sentencias no interrumpidas por otra en contrario, y que estas hayan sido aprobadas por lo menos por cuatro Ministros». Burgoa, 1968: 77-79.

36 Constitución de 1917, artículo 107(I): «La sentencia será siempre tal que sólo se ocupe de individuos particulares, limitándose a ampararlos y protegerlos en el caso especial sobre el que verse la queja, sin hacer una declaración general respecto de la ley o acto que la motivare.»

37 Zamora, Cossío, Pereznieto, Roldán-Xopa y López, 2004: 87-88.

38 Idem.

39 Solicitud de amparo 391A, Manuel Yee, contra prisión y multa de $\$ 100$ bajo la Ley 31, 15 de enero de 1924, CCJSCN, Archivos del Juzgado Quinto de Distrito, Amparo Civil, 1900-1943. Lo que sigue se basa en la información contenida en el archivo del caso Yee. 
un argumento que sirvió también para justificar sus fallos subsecuentes, el juez Espinosa concedió amparo a Yee contra la multa y el encarcelamiento que le había impuesto el presidente municipal de Nogales, Walterio Pesqueira. Apoyado por el agente del Ministerio Público, este juez sentenció que la Ley 31 era claramente anti-constitucional. Violaba la garantía del artículo $1 .^{\circ}$ que aseguraba derechos iguales para todo individuo; contravenía el requerimiento del artículo 13 de que las leyes fueran generales, y la prohibición de ser privado «de la vida, de la libertad o de propiedades, posesiones o derechos» sin un juicio, establecida por el artículo 14.

Según el análisis constitucional del juez Espinosa, al castigar solamente a los chinos, como lo hacía la Ley 31, esta legislación no era de aplicación general, ni establecía derechos iguales. Hizo notar que ni la mancebía ni el concubinato eran crímenes, siempre que las relaciones fueran discretas y no fomentaran el escándalo público. Si estas prácticas debían constituir delitos, tendrían que serlo para todos, y no solamente para los hombres chinos y las mujeres mexicanas con quienes estos establecían relaciones. Además, el juez afirmó que la Ley 31 privaba a Yee de «la vida, de la libertad o de sus propiedades, posesiones o derechos» sin el debido proceso judicial ${ }^{40}$.

Finalmente, el juez Espinosa fincó su fallo directamente en el federalismo. En su opinión, la aplicación de la Ley 31 a Manuel Yee violaba claramente garantías y derechos que proporcionaba la Constitución federal. Citando los principios de supremacía que se establecían en el artículo 133 de la Constitución, Espinosa concluyó que la Ley 31 al ser una norma estatal y no federal, «no pudo servir de fundamento ni ser causa legal» para que un presidente municipal como Pesqueira multara y encarcelara a Yee. Carentes de bases jurídicas, las acciones de Pesqueira eran anti-constitucionales y violaban el requerimiento constitucional del artículo 16 de que solo «la autoridad competente, que funde y motive la causa legal del procedimiento» podía afectar a la persona, o su «familia, domicilio, papeles y posesiones» ${ }^{41}$.

Dada la similitud entre las solicitudes y la rápida sucesión con que se presentaron ante el juez Espinosa, no debe sorprender que este usara el primer fallo como un modelo para los demás. Pareciera que el magistrado redactó una sentencia solo en el caso de Manuel Yee, y la usó como modelo para todas las otras sentencias, puesto que el mismo texto fue escrito una y otra vez, remplazando solo los nombres de los solicitantes. A pesar de que la fórmula Otero estipulaba que la protección otorgada por un amparo no significaba una declaración general sobre la ley o el acto que lo motivase, el primer
40 Idem.
41 Idem. 
amparo que concedió este juez constituyó más que un precedente informal; también fue el texto de las sentencias en los casos subsecuentes.

\section{Julia Delgado y Francisco Gim: La Petición de 1926}

En 1926, Julia Delgado y Francisco Gim acudieron al tribunal federal para ampararse contra la Ley 31. La información básica y los argumentos constitucionales que presentaron ante el juez Joaquín Ruiz eran los mismos que habían hecho Manuel Yee y otros quejosos ante el juez Espinosa en 192442. En su caso, a diferencia del de Yee, el agente del Ministerio Público se opuso al amparo. El juez Ruiz negó la solicitud de Delgado y Gim, rompiendo con el precedente de los casos anteriores.

Tanto Delgado como Gim firmaron la solicitud de amparo que presentaron ante el juez federal Ruiz, reclamando que el juez del Registro Civil en Cananea se había negado a registrar su matrimonio en enero de 1926. Ante esta negativa, Delgado y Gim enviaron un telegrama al gobernador de Sonora, solicitando que autorizara su enlace civil, a pesar de las prohibiciones de la Ley 31. Cuando el gobernador se negó a apoyarlos, Delgado y Gim acudieron ante el tribunal federal alegando que se violaban los artículos 1 y 14 de la Constitución de 1917, como lo habían hecho los solicitantes exitosos en 1924 y 1925 . El texto de la solicitud sostenía la supremacía de la Constitución y de los tribunales federales sobre la legislación discriminatoria del estado de Sonora.

Además de argumentar que la Ley 31 violaba las garantías individuales consagradas en el artículo $1 .^{\circ}$ de la Constitución y contravenía las prohibiciones del artículo 14 de privar a una persona «de la vida, de la libertad o de sus propiedades, posesiones o derechos» sin un proceso judicial adecuado; Delgado y Gim añadieron un reclamo fincado en la retórica de los derechos naturales. Por medio de su abogado, afirmaron que el derecho a casarse era un derecho «que el hombre trae desde que nace» por lo que, «ninguna ley puede quitarle ese derecho máximo» aunque así lo pretendiera la Ley 31.

El agente del Ministerio Público, quien se opuso a esta demanda, respondió con un argumento en tres partes: ponderó los derechos enumerados frente a los no enumerados; los derechos naturales frente al poder del gobierno; y la autoridad de los poderes estatales contra los de la federación. En primer lugar, arguyó, Gim y Delgado no habían identificado con precisión las garan-

42 Solicitud de amparo 12, Francisco Gin y Julia Delgado, aplicación de la Ley número 31, 12 de febrero de 1926, CCJSCN, Archivos del Juzgado Quinto de Distrito, Amparo Civil, 1900-1943. Lo que sigue se basa en la información en el archivo del caso Gin y Delgado. 
tías individuales y constitucionales que atropellaban las acciones del juez del Registro Civil y del gobernador. Como subrayara el agente del Ministerio Público, los derechos enumerados en el artículo 14 de la Constitución - la vida, la libertad, la propiedad, y las posesiones - no incluían específicamente al matrimonio, y Gim y Delgado no habían demostrado de manera convincente que este «derecho» estaba incluido de manera tácita en la enumeración. En segundo lugar, aunque el matrimonio fuera un derecho natural, el gobierno podía limitarlo y protegerlo por medio de la legislación y la regulación. En opinión del Ministerio Público, los beneficios de la discriminación contra chinos que establecía la Ley 31 eran tan obvios que no necesitaban explicación. En tercer lugar, la regulación del matrimonio era facultad del estado de Sonora, y no del gobierno federal. Al no conceder explícitamente la Constitución el poder de regular al matrimonio al gobierno federal, este pertenecía a los estados. Sonora, entonces, podía prohibir el matrimonio entre hombres chinos y mujeres mexicanas si lo consideraba necesario ${ }^{43}$.

La disyuntiva que planteaba reconciliar los derechos naturales con los de nacionalidad, y con las clasificaciones raciales, recorre todos los argumentos esgrimidos en el caso, tanto por los quejosos como por el agente del Ministerio Público y por el juez Ruiz. Por una parte, el abogado de Delgado y Gim enfatizó la naturalización de Gim, pero afirmó que aunque no se hubiera naturalizado, el artículo $1 .^{\circ}$ de la Constitución otorgaba garantías a «todo individuo» y no solo a los ciudadanos o nacionales mexicanos. En cambio, el agente del Ministerio Público insistió que el estatus de Gim, ya fuera extranjero - como incorrectamente lo calificó en un principio- o mexicano naturalizado, no era particularmente relevante. Fueran Gim o incluso Delgado, extranjeros o ciudadanos, la Constitución no protegía ningún derecho a casarse. Desde el punto de vista del Ministerio Público, que el matrimonio fuera derecho natural no tenía relevancia legal, porque no había sido enumerado específicamente en la Constitución. En su fallo, el juez Ruiz se refirió a Gim como «de raza china», sin poner atención alguna en su nacionalidad, fuera la china de origen o a la mexicana por naturalización. En el caso de Delgado, en cambio, el juez Ruiz mencionó su «nacionalidad mexicana». Aunque la sentencia de Ruiz, en la que negaba el amparo a Delgado y a Gim no se basó explícitamente en criterios de raza, nacionalidad, o derechos naturales, las referencias raciales a Gim pero nacionales a Delgado - y el no tomar en cuenta la nacionalidad mexicana de Gim - sugieren que el prejuicio racial influyó en el razonamiento del juez.

\footnotetext{
43 Idem.
} 
Finalmente, y a diferencia de los casos de amparo concedidos anteriormente que presentaban los mismos argumentos, el juez Ruiz falló que el derecho al matrimonio que reclamaban Delgado y Gim carecía de base constitucional. Este magistrado decidió que la Ley 31 había sido promulgada por Sonora «en ejercicio de las facultades concedidas al Estado, como Entidad Libre y Soberana, dentro del régimen representativo, democrático, federal, que la nación se ha dado como sistema de Gobierno» ${ }^{44}$. Regular el matrimonio era responsabilidad y facultad de la entidad federada y no del gobierno federal. Siguiendo la misma línea, Ruiz puso mucho énfasis en el interés del público, esto es, en el interés que tenía Sonora en regular el matrimonio como «medio legal para la formación de la familia, base de la organización social» ${ }^{45}$. Para apuntalar la denegación del amparo, este juez estuvo de acuerdo con el agente del Ministerio Público en que los artículos $1 .^{\circ}$ y 14 de la Constitución no reconocían al matrimonio civil como un derecho natural. En consecuencia, Delgado y Gim quedaron desamparados.

Francisco Gim: LA PETICIÓN DE 1929

A finales de 1929, Gim volvió a pedir amparo contra la aplicación de la Ley 31, esta vez sin la participación explícita de Delgado como quejosa ${ }^{46}$. Aunque Delgado se unió con Gim para solicitar amparo en 1926 cuando el juez del Registro Civil de Cananea se negó a casarlos por lo civil, la mayoría de los recursos de amparo contra la Ley 31 fueron presentados, como el de Gim de 1929, por hombres chinos, sin la participación de sus compañeras mexicanas.

En su petición, como en la de 1926, Gim apuntó que Delgado y él habían procurado casarse por lo civil para «normalizar nuestra vida dentro de los preceptos legales» y para proteger legalmente a la mujer y a sus hijos. En agosto de 1929, como lo habían hecho repetidas veces antes, Gim y Delgado apelaron al gobernador de Sonora para que autorizara su matrimonio. En respuesta, el titular del Poder Ejecutivo del estado ordenó al comisario de policía de Naco imponer las sanciones que marcaba la Ley 31. El 27 de agos-

\footnotetext{
44 Idem.

45 Idem.

46 Petición de amparo 46, Francisco Gin, contra aplicación de la Ley 31, 6 de septiembre de 1929, CCJSCN, Archivos del Juzgado Quinto de Distrito, Amparo Civil, 1900-1943. Lo que sigue se basa en la información en el archivo del caso Gim en 1929 y los documentos de la apelación a la Suprema Corte.
} 
to, el funcionario policial mandó llamar a Gim y le impuso una multa de 200 pesos «por el hecho de estar viviendo en compañía de la señora Delgado» ${ }^{47}$. Cuando el comisario le fijó un plazo de diez días para pagar la multa, Gim apeló a la justicia federal.

La solicitud de Gim de 1929 reveló una estrategia muy distinta a la de 1926. Ya no se refirió a los derechos garantizados en los artículos $1 .^{\circ}$ y 14 de la Constitución, que Delgado había invocado en vano en 1926, aunque esto había resultado eficaz a los otros quejosos. En esta petición, Gim partió de los argumentos del gobierno en el fallo de 1926, los mismos que habían servido para negarle amparo, fincando su argumentación en los principios del federalismo y de la división constitucional de poderes entre el gobierno federal y los estatales. Gim dio a estos argumentos una dimensión internacional.

Por medio de su abogado, Gim arguyó que el artículo 73(XVI) de la Constitución reservaba al Congreso federal todo el poder de legislar sobre los extranjeros y sus intereses. De manera similar, el artículo 31 de la Ley de Extranjería y Naturalización de 1886 establecía que solo la ley federal podía modificar o restringir los derechos civiles de que gozaban los extranjeros. Por lo tanto, la ley federal era superior a la Ley 31 y, en esencia, aquella derogaba a esta por representar una política estatal que pretendía limitar el derecho de los extranjeros a casarse. A nivel internacional, Gim se refirió al Tratado de Amistad, Comercio y Navegación entre México y China, firmado en 1899. Este Tratado, afirmaba Gim, establecía reciprocidad entre los ciudadanos chinos residentes en México y los ciudadanos mexicanos. Citó la cláusula del Tratado que afirmaba que «los ciudadanos chinos en México gozarán de los mismos derechos y concesiones de que gozan los mexicanos o los súbditos de la nación más favorecida» ${ }^{48}$. Gim resumió así los argumentos basados en el federalismo y el derecho internacional: "si la mencionada Ley número 31 contiene disposiciones contrarias a las demás que se acaban de citar [incluyendo el Tratado con China], viola las garantías individuales o por medio de ella el Congreso Local invadió la esfera de la autoridad federal» ${ }^{49}$. Estos dos argumentos - que la ley federal tenía primacía sobre la legislación estatal y que un tratado internacional obligaba reciprocidad para los ciudadanos chinos - giraban sobre la hipótesis de que Gim era extranjero, no mexicano.

\section{Idem.}

48 Idem. Aunque el gobierno federal canceló el tratado de 1899 con China en octubre de 1927, parece que algunos abogados en Sonora, incluyendo al abogado de Gim, no lo sabían, pues lo trataban como ley vigente en las solicitudes de amparo todavía en 1929.

49 Idem. 
A renglón seguido, Gim formuló un argumento alternativo: él era nacional de México, no extranjero. Había llenado todos los requisitos especificados en el artículo 30(II-B) de la Constitución para naturalizarse; por lo tanto, tenía derecho a todas las protecciones y garantías que otorgaba la Constitución, particularmente el artículo 16, que especificaba que nadie podía «ser molestado en su persona, familia, domicilio, papeles y posesiones» sino tras un proceso legal adecuado. Afirmaba también que, al aplicarse en su caso la Ley 31, se contravenía el artículo 29 de la Ley de Extranjería y Naturalización de 1886, que dotaba a los mexicanos por naturalización con todos los derechos y deberes que tenían los mexicanos por nacimiento.

El abogado de Gim formuló dos argumentos más sobre el proceso y la autoridad en la solicitud de amparo de 1929: 1) la multa de 200 pesos que había impuesto a Gim el comisario de policía excedía el límite de quince pesos que la Ley Orgánica de Sonora establecía a los gravámenes que podían imponer las autoridades municipales; y 2) la multa de 200 pesos era en realidad una pena, y estas solo se podían imponer constitucionalmente, siguiendo los procedimientos judiciales que describía el artículo 21 de la Constitución ${ }^{50}$.

Al final, el juez Silva falló que la Ley 31 y las sanciones que impuso el comisario de policía no violaban ningún derecho o garantía otorgados a Gim por la Constitución, el artículo 29 de la Ley de Extranjería y Naturalización de 1886, o el proceso judicial. El juez Silva negó amparo a Gim. En una acción bastante agresiva y atípica de los solicitantes de amparo chinos, el 9 de septiembre de 1929, Gim apeló ante la Suprema Corte de la Nación.

\section{La Suprema Corte}

La apelación de Gim desafiaba, punto por punto, la sentencia del juez Silva presentando once agravios. Estos exponían los errores que supuestamente había cometido el juzgado de primera instancia, cuya sentencia afirmaba que Gim había accedido a la aplicación de la Ley 31 y que dicha ley no invadía facultades federales al regular a los extranjeros. La apelación de Gim finalizó en el mismo tono con que había concluido la solicitud original, subrayando los argumentos técnicos sobre la falta de autoridad del comisario de policía para imponer una multa. Gim amplió sus alegatos para hacer notar que la Ley Orgánica de Sonora distinguía entre las autoridades municipales y la

50 Petición de amparo 46, Francisco Gin, contra aplicación de la Ley 31, 6 de septiembre de 1929, CCJSCN, Archivos del Juzgado Quinto de Distrito, Amparo Civil, 1900-1943. 
policía: la policía no era autoridad municipal. Entonces, no solo la multa de 200 pesos excedía el límite autorizado por la Ley Orgánica de Sonora, sino que esta no autorizaba al comisario a imponerla.

El agente del Ministerio Público Federal, designado por el Procurador General para intervenir en el caso, apoyó la apelación de Gim$^{51}$. El agente puso más énfasis en el federalismo como base para conceder el amparo. En primer lugar, la Ley 31 regulaba derechos de extranjeros, facultad reservada al gobierno federal por el artículo 73 de la Constitución. En segundo lugar, aun si pudiera reconciliarse la Ley 31 con el artículo 73, aquella violaba los derechos constitucionales y naturales de Gim como mexicano por naturalización. El Ministerio Público insistió en el poder transformativo de la ley federal de naturalización, el mismo que negaba Sonora. La naturalización de Gim garantizaba su derecho fundamental a «la constitución de la familia, base de la Sociedad Mexicana ${ }^{52}$. El negarle el derecho de casarse, y, aun peor, imponerle por ello una multa, infringía «derechos inalienables que solamente pueden ser modificados o restringidos por una disposición del Congreso de la Unión, más nunca por una Ley de un Estado que, en todo caso, debe estar supeditada a las leyes federales $\rangle^{53}$. La ley federal era suprema, y por tanto amparaba a Francisco Gim contra la Ley 31.

La Segunda Sala de la Suprema Corte de Justicia estuvo de acuerdo, pero no por las razones que esgrimió el Ministerio Público. Por medio de una decisión redactada por el magistrado Luis Calderón, aquella Sala amparó a Francisco Gim el 23 de septiembre de $1930^{54}$. La base de la sentencia fue el punto técnico y de proceso que el abogado de Gim había añadido al final de la petición inicial. La Sala falló que, bajo los requisitos del artículo 21 de la Constitución, la imposición de penas pertenecía a la judicatura. La Ley 31 permitía a autoridades no judiciales imponer penas y en los hechos, autorida-

51 La segunda Ley Orgánica de 1919 asignó a la Procuraduría General «intervenir en todos los juicios de amparo». La segunda Ley Orgánica esta disponible en http://www.pgr. gob.mx/que\%20es\%20pgr/historia.asp. El agente en este caso indica que «fue designado por el Procurador General de la República para intervenir en el amparo en revisión promovido por Francisco Gin». Petición de amparo 46, Francisco Gin, contra aplicación de la Ley 31, 6 de septiembre de 1929, CCJSCN, Archivos del Juzgado Quinto de Distrito, Amparo Civil, 1900-1943.

52 Idem.

53 Idem.

54 Eran miembros de la Segunda Sala, el presidente Arturo Cisneros Canto y los magistrados Salvador Urbina, Jesús Guzmán Vaca, Daniel Valencia y Luís M. Calderón. Vease Petición de amparo 46, Francisco Gin, contra aplicación de la Ley 31, 6 de septiembre de 1929, CCJSCN, Archivos del Juzgado Quinto de Distrito, Amparo Civil, 1900-1943. 
des no judiciales habían impuesto una pena de 200 pesos a Gim. Por esto, la Segunda Sala votó de forma unánime amparar a Gim contra la multa y ampararlo, por tres votos contra dos, contra la Ley 31. Sin embargo, en este amparo, pasó a segundo plano la solicitud explícita de Gim para que se autorizara su matrimonio con Julia Delgado. En el fallo de la Corte, no se dirigió ninguna orden al juez del Registro Civil de Naco o al gobernador del estado de Sonora para que permitieran el casamiento de Delgado y Gim, o para que se registrara su matrimonio. Se amparó a Gim, pero Delgado y los hijos de ambos no recibieron las protecciones legales que debía proporcionar el matrimonio civil.

Carlos Wong Sun: La Petición de 1929

Cuando Francisco Gim apeló a la Suprema Corte en octubre de 1929, este tribunal tenía pendiente otra solicitud presentada en junio de 1929 por Carlos Wong Sun ${ }^{55}$. Aunque había llegado antes a la Corte, el caso de Wong Sun no se resolvió sino hasta diciembre de 1932, más de dos años después de que la Corte amparara a Gim.

Estos dos casos fueron los únicos recursos interpuestos ante la Suprema Corte contra la Ley 31, y eran a la vez similares y distintos. Ambos solicitaron amparo en 1929 ante la negativa de funcionarios del Registro Civil de Sonora de permitir el casamiento con una mujer mexicana. El juez federal de distrito, Joaquín Silva, negó a ambos el amparo, aunque los fallos fueron diferentes porque lo eran también los hechos. El juez del Registro Civil negó a Gim licencia para casarse y le impuso una pena monetaria. Wong Sun había mantenido su ciudadanía china mientras que Gim se había naturalizado. Además, Arsenio Espinosa, el antiguo juez federal que había amparado a tantos chinos contra la Ley 31 en 1924 y 1925, ahora era el abogado de Wong Sun.

Al elaborar la solicitud de amparo de Wong Sun, Espinosa presentó los mismos argumentos sobre los que había basado sus fallos judiciales a favor de los chinos cinco años antes: la Ley 31, en su texto y en su aplicación, violaba los artículos $1 .^{\circ}, 13,14$, y 16 de la Constitución federal. La provisión en el artículo 1..$^{\circ}$ de «que todos los habitantes de la República Mexicana gozarán de las garantías» quería decir que «todos los residentes de México»,

55 Solicitud de amparo 10, Carlos Wong Sun, contra aplicación de la Ley 31, 10 de febrero de 1929, CCJSCN, Archivos del Juzgado Quinto de Distrito, Amparo Civil, 1900-1943. Lo que sigue se basa en la información en el archivo del caso Wong Sun y los documentos de la apelación a la Suprema Corte. 
como Wong Sun, «tienen los mismos derechos civiles y gozan de las mismas garantías individuales» que los ciudadanos mexicanos ${ }^{56}$. Además, el código civil de Sonora establecía las condiciones y los impedimentos para el matrimonio, sin mencionar la raza. Al hacerlo así, el código simplemente reconocía formal y legalmente «un derecho natural de todo ser humano» ${ }^{57}$. Por restringir el derecho a casarse de los chinos, la Ley 31 violaba el artículo $1 .^{\circ}$ y, por tanto, los derechos naturales de Wong Sun, aunque Espinosa no insistió en este punto. Del mismo modo, el texto de la Ley 31 violaba el requerimiento del artículo 13 de que las leyes se aplicaran de manera general y no exclusivamente a una comunidad como la china.

Cuando el juez del Registro Civil en Cucurpé aplicó la Ley 31 y se rehusó a dar permiso a Wong Sun de casarse, había contravenido el artículo 14, que prohibía el privar a cualquiera de «la vida, de la libertad o de sus propiedades, posesiones o derechos» sin un proceso judicial. Sus acciones también iban contra del artículo 16, que estipulaba que solo las autoridades competentes podían restringir derechos o interferir con un individuo o la familia. El único argumento que faltaba en el análisis de Espinosa era el del federalismo que como juez había esgrimido, afirmando la supremacía de la Constitución que aseguraba que sus disposiciones prevalecerían sobre leyes estatales que les fueran contrarias, como era el caso de la Ley 31.

Aunque Espinosa hubiera incluido al federalismo para apuntalar la solicitud de Wong Sun, probablemente no hubiera persuadido al juez Silva. Este afirmó que el matrimonio era un derecho natural, pero en el campo legal no representaba más que «un contrato de sociedad legítima» ${ }^{58}$. La constitución federal no regulaba el matrimonio, y por lo tanto ese poder y responsabilidad quedaban en manos de los estados, los cuales podían, «en ejercicio de su soberanía», poner los términos y condiciones a los contratos civiles que consideraran convenientes ${ }^{59}$. La aplicación de la Ley 31 a Wong Sun no violaba el artículo 14 porque «aunque es verdad que dicho señor tiene el derecho de contraer matrimonio, no es menos cierto que no puede ejercitar ese derecho en el Estado de Sonora, sino con sujeción a las disposiciones emanadas del Congreso Local que regulan dicho contrato» ${ }^{60}$.

De manera concluyente, el juez Silva falló que la Ley 31 no violaba los artículos $1 .^{\circ}, 13 .^{\circ}$ y $16 .^{\circ}$ de la Constitución federal. El artículo 1 no «consagra
56 Idem.
57 Idem.
58 Idem.
59 Idem.
60 Idem. 
garantía individual alguna en concreto» y menos el derecho a casarse con una mujer mexicana ${ }^{61}$. Como no se trataba de un proceso judicial, Wong Sun no podía reclamar que se le aplicaban leyes privativas, contraviniendo el artículo 1362. El negar el derecho a casarse simplemente no violaba el artículo $16^{63}$.

Wong Sun apeló el fallo del juez Silva ante la Suprema Corte en junio de 1929. Su alegato concedía que los estados pudieran regular el contrato de matrimonio, pero argüía que el poder de «fijar las condiciones, forma y alcance de tal contrato» no significaba prohibir su celebración ${ }^{64}$. Al prohibir la Ley 31 que se casaran hombres chinos con mujeres mexicanas, negaba el derecho natural al matrimonio, en contravención del artículo 14 constitucional. Del mismo modo, la apelación condenó el fallo del juez Silva basándose en los artículos 1. ${ }^{\circ}, 13$ y 16 y alegando que los derechos naturales formaban parte del patrimonio nacional, privar a Wong Sun de su derecho a casarse interferiría con sus derechos constitucionales.

Este recurso estuvo demorado en la Suprema Corte por más de tres años. Cuando la Segunda Sala por fin falló en diciembre de 1932, el magistrado Daniel Valencia afirmó contundentemente que el artículo 130 de la Constitución de 1917 daba «al matrimonio el carácter de contrato civil» ${ }^{65}$. Por ser el matrimonio un contrato civil y no una garantía individual, Sonora tenía «plena soberanía para legislar sobre la materia» y podría prohibir el matrimonio entre mujeres mexicanas y hombres chinos si lo juzgaba conveniente ${ }^{66}$. La prohibición del mestizaje entre chinos y mexicanas que establecía la Ley 31 era simplemente «un impedimento más» a la celebración del matrimonio en Sonora ${ }^{67}$. El magistrado Valencia razonó que la ley no privaba a Carlos Wong Sun de ningún derecho, de ninguna garantía, porque no tenía derecho a contraer matrimonio en primer lugar ${ }^{68}$. El regular el matrimonio competía a los estados; una ley contra el mestizaje no violaba las garantías de la Constitución federal. Carlos Wong Sun quedó desamparado.

Los tres ministros de la Segunda Sala - Arturo Cisneros Canto, Daniel Valencia y Luis Calderón - que votaron por amparar a Francisco Gim contra
${ }^{61}$ Idem.
62 Idem.
63 Idem.
64 Idem.
65 Idem. La segunda sala se componía del presidente Luis M. Calderón y los ministros Arturo Cisneros Canto, José López Lira, Daniel V. Valencia y Jesús Guzmán Vaca. El magis- trado Guzmán Vaca estuvo ausente el día en que la sala decidió el caso Wong Sun. Idem.
66 Idem.
${ }^{67}$ Idem.
68 Idem. 
la multa y de la Ley 31, formaban también la mayoría en el caso de Wong Sun ${ }^{69}$. En este caso, sin embargo, los tres votaron por negar el amparo, al igual que el cuarto ministro, José López Lira. Los magistrados no explicaron por qué cambiaron de opinión entre septiembre de 1930 y diciembre de 1932, ni hicieron referencia al caso Gim. Aun dadas las limitaciones de la fórmula Otero para tomar otros casos como precedentes, es sorprendente que no se cite el caso Gim, dada su proximidad en el tiempo y el hecho de que fueran las únicas apelaciones a fallos sobre la Ley 31 que oyera la Corte.

\section{Conclusión}

Al presentar solicitudes de amparo, parejas de mexicanas y chinos recurrieron a la ley federal para afirmar sus derechos constitucionales cuando el estado de Sonora estableció la discriminatoria Ley 31. El recurso al amparo por parte de los afectados en la Suprema Corte y en los tribunales federales de primera instancia pone de manifiesto los desafíos y las oportunidades que proporcionaban la ley y el derecho a ciertos sectores marginados en México en el primer tercio del siglo XX.

En los dos casos sobre la Ley 31 ante la Suprema Corte de la Nación, la Segunda Sala basó su decisión en las atribuciones de las autoridades involucradas dentro del marco del federalismo; en el caso de Francisco Gim, la pregunta era si los funcionarios municipales eran competentes para imponer penas y multas, y en el caso de Carlos Wong Sun si podía el estado regular el matrimonio. La Segunda Sala fundamentó sus fallos en el federalismo, y no en los derechos constitucionales. Aprobó la discriminación legal en contra de los chinos en Sonora. Al hacer esto, contravino las promesas de igualdad y libertad que enunciaba la Constitución de 1917.

En contraste, en los tribunales federales de primera instancia, el éxito casi completo de los chinos que pidieron amparo contra la Ley 31 en 1924 y 1925 representa un momento breve en la historia del derecho en México en el que un grupo pequeño de jueces federales afirmó estas promesas de igualdad y libertad para una minoría despreciada. Los jueces federales, y Arsenio Espinosa en particular, hicieron esto aplicando estrictamente la ley, afirmando la supremacía de la Constitución Federal sobre la legislación ordinaria del estado de Sonora. La fórmula Otero negaba el valor de precedente formal a los fallos judiciales, y la Suprema Corte rechazó fundamentar sus fallos en las expectativas que generaba la Constitución. Sin embargo, los procesos de am-

\footnotetext{
69 Idem.
} 
paro contra la Ley 31 que oyó el juez Arsenio Espinosa, fallando a favor de los quejosos, representan un ejemplo esperanzador y brillante de la interpretación constitucional en México.

\section{REFERENCIAS CONSULTADAS}

\section{ARCHIVO}

CCJSCN. Casa de la Cultura Jurídica de la Suprema Corte de la Nación, Hermosillo, Sonora, México.

\section{BiBLIOGRAFÍA}

Augustine-Adams, Kif, «Making Mexico: Legal Nationality, Chinese Race, and the 1930 Population Census», Law and History Review, XXVII / 1 (Illinois, USA, 2009): 113-44.

Burgoa, Ignacio, El juicio de amparo, México, D.F, Porrúa, 1968.

Espinoza, José Angel, El ejemplo de Sonora, México, s.e., 1932.

González Oropeza, Manuel, «La discriminación en México: El caso de los nacionales chinos», Cuadernos del Instituto de Investigaciones Jurídicas. La problemática del racismo en los umbrales del siglo XXI, VI Jornadas Lascanianas, México, D. F., UNAM, 1997: 47-56, disponible en http://www.bibliojuridica.org/libros/1/ 148/5.pdf.

Hale, Charles A., «The civil law tradition and constitutionalism in twentieth-century Mexico: The legacy of Emilio Rabasa», Law and History Review, XVIII / 2 (Illinois, USA, 2000): 257-280.

Hale, Charles A., Emilio Rabasa and the survival of porfirian liberalism, Stanford, California: Stanford University Press, 2008.

Hu-Dehart, Evelyn, «La comunidad china en el desarrollo de Sonora», Historia General de Sonora, tomo IV: Sonora Moderno 1880-1929, Hermosillo, Sonora, Gobierno del estado de Sonora, 1985: 195-211.

Liam, Julian «Chinos and Paisanos: Chinese Mexican Relations in the Borderlands», Pacific Historical Review, LXXIX / 1 (California, 2010): 50-85.

Mirow, M.C., «Marbury in Mexico: Judicial review's precocious southern migration», Hastings Constitutional Law Quarterly, XXXV / 1 (Hastings, California 2007-2008): 41-117. 
Réñique, Gerardo, «Región, raza y nación en el antichinismo sonorense: Cultura regional y mestizaje en el México posrevolucionario», A. Grageda Bustamante (ed.), Seis expulsiones y un adiós: Despojos y exclusiones en Sonora, México, Plaza y Valdés, 2003: 231-89.

Salyer, Lucy, Laws harsh as tigers: Chinese immigrants and the shaping of modern immigration law, North Carolina, USA, University of North Carolina, 1995.

Schiavone Camacho, Julia Maria, «Crossing Boundaries, Claiming a Homeland: The Mexican Chinese Transpacific Journey to Becoming Mexican, 1930s-1960s», Pacific Historical Review, LXXVIII / 4 (California, 2009): 545-577.

Suprema Corte de Justicia de la Nación, Ministros 1917-1994: Semblanzas, dirección general de estudios históricos, t. I, México, Suprema Corte de Justicia de la Nación, 2002.

Supreme Court of the United States, «The Chinese Exclusion Case», United States Reports, 130 (Washington, 1889): 581-611.

Supreme Court of the United States, «Yick Wo v. Hopkins», United States Reports, 118 (Washington, 1886): 356-374.

Supreme Court of the United States, «Marbury v. Madison», United States Reports, 5 (Washington, 1803): 137-139.

Vallarta, Ignacio Luís, Cuestiones constitucionales: Votos que como presidente de la Suprema Corte de Justicia dio en los negocios más notables resueltos por este tribunal de 1 de enero a 16 de noviembre de 1882, t. 3, México, D.F.; n.p. 18811883.

Zamora, Stephen, Cossío, José Ramón, Pereznieto, Leonel, Roldán-Xopa, José y López, David, Mexican law, Oxford, England, Oxford University Press, 2004.

Fecha de recepción: 24 de mayo de 2011

Fecha de aceptación: 10 de febrero de 2012 


\section{Prohibiting miscegenation with the Chinese: «Amparo» petitions, Sonora, 1921-1935}

In the state of Sonora, Mexico, at the beginning of the Twentieth Century, the Chinese population faced vicious discrimination including Law 31. Promulgated by the state legislature in late 1923, Law 31 prohibited marriage and other intimate relations between Chinese men and Mexican women. Through amparo petitions in federal court, up to and including the Supreme Court of Mexico, Chinese Mexican couples challenged the discrimination inherent in Law 31. Chinese Mexican amparo petitions against Law 31 demonstrate both the challenges and possibilities that law provided to the disenfranchised in Mexico at the beginning of the Twentieth Century.

Key words: Mexico; Chinese; discrimination; Matrimonial Unions; Legal Processes. 